\title{
Combating STI Epidemic: Community Perspectives Learned from Mixed-Gender Focus Group Study
}

\author{
K. M. Islam, ${ }^{1}$ Laura Vinson, ${ }^{2}$ Sherri Nared, ${ }^{3}$ Ira Combs, ${ }^{4}$ Melissa Tibbits, ${ }^{5}$ \\ Shinobu Watanabe-Galloway, ${ }^{6}$ and Ruth Margalit ${ }^{7}$
}

${ }^{1}$ Department of Epidemiology (Dept. Epi), College of Public Health (CoPH), 984395, University of Nebraska Medical Center (UNMC), Omaha, NE 68198-4395, USA

${ }^{2} \mathrm{CoPH}, \mathrm{UNMC}$, USA

${ }^{3}$ Douglas County Health Department, NE, USA

${ }^{4}$ Center for Reducing Health Disparity, CoPH, UNMC, USA

${ }^{5}$ Department of Health Promotion, Social \& Behavioral Health, CoPH, UNMC, USA

${ }^{6}$ Dept. Epi, CoPH, UNMC, USA

${ }^{7}$ Department of Health Promotion, Social \& Behavioral Health and Director, Inter-Professional Service Learning Academy, CoPH, UNMC, USA

Correspondence should be addressed to KM Islam; kmislam@unmc.edu

Received 28 March 2013; Accepted 28 April 2013

Academic Editors: R. Andersson, R. Favory, T. Hazir, and T. Matsumoto

Copyright $\odot 2013$ K. M. Islam et al. This is an open access article distributed under the Creative Commons Attribution License, which permits unrestricted use, distribution, and reproduction in any medium, provided the original work is properly cited.

Background. Since 2004, Douglas County in Nebraska has been experiencing higher rates of sexually transmitted infections (STIs) than the rest of the state. Northern Omaha has been considered to be one of the "hot spots" for STIs in the county. In 2011-2012, a focus group study was conducted to investigate the community's perception of STIs and to identify strategies to increase the testing and treatment of STIs. Methods. Fourteen young adults were recruited to participate in two mixed-gender focus group sessions, each lasting 1.5 hours. Sessions covered various topics on STIs. Audio-recorded sessions were transcribed and analyzed by the study team. Results. Participants were aware of the STI problem in their community. They identified risk factors for STIs included careless sexual behavior, unprotected sex, and lack of communication with parents. Participants believed that the club environment and drug and alcohol use contribute to risky sexual behavior. Participants perceived lack of confidentiality as the most important barrier to getting tested. The most important reason for not seeking treatment was lack of awareness about the signs of infection. Participants called for measures to increase trustworthy relationships with the health care system and suggested opening more testing and treatment sites. Discussion. The uniqueness of our study is that it is the first conducted epidemiological study in this population to better understand risk factors of STIs specific to this population. The study identified several important factors that may assist in future interventions to reduce STIs in this population.

\section{Background}

Sexually transmitted infections (STIs) are a major public health problem in the Omaha, Nebraska. Since 2004, rates of STIs in Douglas County have been consistently above national and state rates, with estimated rates of Gonorrhea approaching twice the rates for Nebraska and the United States [1]. In 2010, rates of Chlamydia trachomatis (CT) were 527.5 per 100,000 populations in Douglas County, compared to 305.2 per 100,000 in Nebraska and 409.2 per 100,000 in the United States. During the same year, the rate of Neisseria gonorrhea (NG) were 186.6 per 100,000 in Douglas County, compared to 77.2 per 100,000 in Nebraska and 99.1 per 100,000 in the United States [2]. A Douglas County Health Department (DCHD) report for 2010-2011 showed that more than $70 \%$ of CT cases and $61 \%$ of NG cases were among adolescents (15-19 years old) and young adults (20-24 years old). In addition, data showed African Americans (AAs) were 
disproportionately affected; $44 \%$ of CT cases and $64 \%$ of NG cases were identified in AAs, though they make up only $12 \%$ of the county's population [2].

CT and NG often go undetected and untreated because many individuals, especially women, have only mild or no symptoms [3]. If untreated, CT can cause serious short- and long-term reproductive and other health consequences [4], including pelvic inflammatory disease (PID), with permanent damage to the fallopian tubes, uterus, and surrounding tissues, which may lead to infertility, ectopic pregnancy, or premature delivery [5]. CT is a leading cause of infant pneumonia, and NG can cause blindness or a life-threatening blood infection in the baby $[6,7]$. Furthermore, individuals infected with CT or NG is at higher risk for HIV infection $[6,7]$. It is clear that the STI epidemic in Douglas County is a serious public health problem.

Though the STI rates in Douglas County have been in epidemic proportions since 2004, reasons for persistently high rates are not clearly understood. The problem is likely a result of a complex interplay of multiple factors. To date, epidemiological studies have not been conducted to investigate factors that put residents of Douglas County, particularly AAs young adults, at higher risk for contracting and sustaining STIs. Identifying factors that place residence of northern region of Douglas County at higher risk for contracting STIs, as well as factors that contribute to the continuation of high incidence rates of STIs in the population can inform the development of appropriate interventions.

\section{Study Methods}

Focus groups were conducted to gain insight and understanding of risk factors, testing resources and treatment options for STIs in the northern region of Douglas County. Two focus group sessions were conducted, 1.5 hours each with study participants 19 to 25 years old. Given the high prevalence of CT and NG among AAs youth in the Northern part of Douglas County, we limited enrollment to youth from the Northern region of the county. The structure of the focus groups allowed for more in-depth discussion of issues related to STIs, compared to what would have been possible with a survey. The use of a trained moderator was essential to create a safe open environment and to ensure that all persons had an opportunity to share their perceptions and comment on other's perceptions. The moderator also used probing phrases and questions to help facilitate the discussion and encourage participants to expand on their perceptions. Focus groups generally work best with 7-9 participants in each group [8]. Our target was AAs living in the northern region of Douglas County.

The project team sought for suggestions from the community members about the composition of the focus groups. Mixed-gender focus groups were identified as a preferred composition. It was suggested that opportunities would be provided for each gender to respond to the other's perceptions, allowing a more in-depth discussion and varied responses.
Participants for the focus group sessions were recruited by several community leaders and health care professionals working in the North Omaha community. Facilitators were experienced interviewers, were involved in the design of the study, and were knowledgeable about the specific aims of the study, the process of informed consent, study ethics, and study safety. The study team utilized a chain-referral sampling recruitment method; community leaders identified potential participants for the study and referred them to verify their eligibility [9].

Those who were eligible to participate were then asked to refer additional potential participants for the study. Multiple sites and community leaders were used for recruitment to ensure a wide coverage of the population, giving a representative sample for this study. The topics in the first part of the focus groups included (1) STDs in the community, (2) STD risk factors, and (3) STD testing- and treatmentrelated issues. The topic in the second part of the focus groups focused on participants' suggestions for appropriate approaches for STI testing in this community.

2.1. Demographics of Focus Group Participants. A total of 14 participants were involved in the two sessions, including 9 males and 5 females. The mean age was 22.3. The distribution of race was 12 black and 2 white. The participant's occupation was categorized as student $(n=6)$, employed $(n=4)$, and unemployed $(n=2) ; 2$ participants did not report occupation. The participants' education level was categorized as high school graduate $(n=6)$, some college $(n=4)$, and college student $(n=3)$; 1 participant did not report education information. The participants' geographic distribution was categorized by ZIP codes in North Omaha: $68111(n=6)$, $68110(n=1), 68104(n=3), 68152(n=3)$, and 68105 $(n=1)$. Most participants were single $(n=13)$, but one participant did not report marital status. Four of the participants reported that they had children, two participants did not answer the question, and eight participants reported that they did not have children.

2.2. Analysis of Information Collected. Audio-taped focus group sessions were transcribed from the recordings, and through an iterative process, the research team identified key themes from the notes. Three reviewers compared those selected common themes and phrases from each focus group session to develop a summary document. From this document the final summary report was developed to include exact quotations supporting the summary phrases.

\section{Results}

For each of the three question topics in Part 1, there were up to four follow-up questions (the appendix). The purpose of these questions was to understand the participants' perceptions of the burden of STIs, its risk factors, and barriers to screening and treatment options in this community.

3.1. Perceived Burden of STIs. Overall, participants were aware of the STI problem. Participants acknowledged that 
STIs are a major public health problem. They also recognized that not enough is done to address this in their community. They explained the high STI rates with reasons such as sex starting at a younger age 9 (younger than participants), sex with multiple partners, and poor sex education from both parents and schools. Participants blamed "younger kids" for the problem (participants referred younger than 19 years old), refusing to take responsibility for the high STI rates in their community. This finding may imply that young adults are not ready to take responsibility for problems in their community at this time. One participant said, "Small town-everyone knows everyone else." By this, the participant meant that because the North Omaha community is so small, there are few choices for new sexual partners, and the spread of STIs is greater.

3.2. Risk Factors for STIs. When asked about the risk factors for STIs in their community, participants' responses pointed to a wide range of factors, including careless behavior in youth, lack of knowledge about STIs, sexual intercourse without protection, the common practice of experienced partners having sex with virgins, establishment of social status by sleeping with someone "important" in the community, the popularity of having sex with more experienced partners, and the club environment, which promotes casual unprotected sex.

Youth engage in sexual activities with multiple partners, do not care who is sleeping with whom, and want to have sex at the moment. Some participants suggested that youth have multiple partners intending to infect others by "passing it on to" others. The participants also reflected that youth believe they are invincible to STIs; therefore, they are careless in regard to sexual behavior. Participants shared that youth are influence by the media and that they think the community is small, with every youth having sex with everyone else.

The club environment was referred to by participants as "booty shaking, skimpy clothing, and being drunk," an environment that places individuals at higher risk for engaging in risky sexual activities. Additionally, the lack of communication with parents, as well as fear of the outcome (unknown factors) keeps individuals 19-25 years old from testing or being screened for STIs. The behaviors discussed above are known to be risk factors for higher transmission of STIs in the community.

Participants unanimously identified abstinence, use of condoms, and protection (other than condoms) as measures to avoid getting STIs. They also emphasized the importance of a monogamous sexual partner, education, increased STI testing, reduced alcohol use, and increased access to reliable condoms.

Participants believed that social, environmental, and behavioral risk factors contribute to the epidemic. Again, the club environment topped the list among risk factors, followed by drug and alcohol use. Participants reported that peer pressure, a carefree environment, and the perception of invincibility, among other risk factors, are responsible for the high STI rates. Participants also noted that individuals were not getting tested because of a perceived lack of trust and confidentiality in health care systems. Participants were most concerned about privacy and confidentiality. They reported that they would travel to the other side of the town to maintain privacy. They also believed that their confidentiality may not be maintained by the health care system. This finding is similar to that in an earlier study on staff and client perceptions of quality of care at a sexually transmitted disease clinic [10]. Focus group participants also mentioned that living in certain environmental conditions and/or geographic areas can put someone at higher risk; for example, the club environment and being drunk can lead to a "jumping-off point." The "jumping-off point" was explained as the point before someone makes a decision, after which the person is "wrapped up and don't care about consequences and you do whatever you want." One female participant noted that in the North Omaha community, it is almost impossible for a female to ask a male partner to use a condom. She said, "As a female, I don't feel it's appropriate to initiate condom use prior to sex if the male doesn't have a condom."

3.3. Barriers to Screening and Treatment. Participants were knowledgeable about the STI screening and treatment facilities in Omaha; however, many struggled to name specific adequate resources in their own geographic community Participants could typically name one facility for screening and treatment, but the majority of the responses were either outside of the North Omaha community or facilities that have moved. One participant expressed his/her frustration by saying, "Nothing is here in North Omaha." (What is often referred to as "health desert" by the community members).

Participants were asked, "Why don't some people get tested for STDs?" The overwhelming response was "confidentiality" - peers and parents may find out, and fear of being judged, especially a major concern for females, and fear to know the truth (the disease and its unknown outcomes). Feeling embarrassed and considering it as a private issue were also reasons to not get tested. Another reason for not getting tested was because most of the time individuals did not know they had a STI due to the lack of symptoms. Others thought they cannot get a STI because they are invincible. The location of testing sites was also an impediment for testing of STIs. Males were not worried about the location of testing sites, if they had peer support with them. Other competing facts of life are another big obstacle for testing, according to one participant, "too busy." More specific reasons related to the African American community were a sense of judgment and the associated stigma as major factors for not getting tested for STIs.

Participants reported that individuals do not get treatment for STIs because they do not get tested and so they do not know they have an STI. Fear plays a major role for not being tested and getting treatment for STIs. However, a participant shared that this as a different kind of fear, "hopes and dreams go down the drain." Some are scared to get treatment due to the misconceptions about the options of treatment. Also there is a perception about the timing of the treatment and the outcome of the treatment, as one participant reflected that "damage has been done already." 
Some are afraid of being social outcasts. Participants shared that there are a few who want to intentionally pass it on to others (I have it and you should have it too). Finally, participants felt it was expensive to get treatment for STIs.

3.4. Suggestions on Testing and Treatment. Participants provided a wide range of ideas to increase testing and treatment for STIs in North Omaha. They suggested more advertisement of STIs such as billboards, and giving STIs a "face," meaning to have a billboard with a picture of a famous person (such as a movie star with whom residents are familiar) admitting they had an STI. Furthermore, the billboard could show the famous person explaining that due to screening, doctors caught the STI early and cured it. Participants also asked that more places for testing and treatment be available, including home visits, a mobile STI unit, or pullup locations not in their own neighborhood (not a clinic). They emphasized education about STIs in schools, starting at age 13 , and requiring a human growth class. They suggested that peers promote testing (good role models), that testing be offered at major events such as hip hop concerts, and that incentives be offered (e.g., get paid if you get tested). Finally, participants reported that making testing and treatment free, with better confidentiality assurance, could increase testing and treatment for STIs in the North Omaha community.

Participants suggested that existing venues are a viable option for STI testing if locations are appropriate, such as at a community center, but not at a basketball game. However, participants said that incentives such as food, money, and socializing are needed to induce people to be tested. Participants said that testing during a home visit would work, but only if an appointment was scheduled prior to the health care worker meeting/coming to a person's house. There were no responses regarding the pros and cons of offering STI testing at neighborhood clinics or at a doctor's office. Participants offered other suggestions for STI testing in their community, "something you can pick up and send in, but with simple instructions," for example, a urine sample collection via the US Postal Service; urine samples collection centers, and a call center to set up appointments. Finally, the participants advised establishing good public relations and incentives and/or benefits for a successful STI testing program.

\section{Discussions}

The focus groups suggested that participants' have limited knowledge about STIs, available services, treatment cost, and beliefs regarding stigma are barriers to screening and treatment. These findings are consistent with the literature [11].

Our study is the first epidemiological study conducted in the Northern Omaha community to better understand the risk factors specific to this population. The study's findings point to several factors that are unique to this population, such as sex with virgins, sex with someone who is important in the community, and sex with experienced partners, all of which are factors similar to those of intergenerational sexual partnerships. Such relationships may be a major source of transmission of common STIs to young and previously virgin populations in this community. Participants suggested that individuals may have as many as eight sexual partners concurrently, which is surprisingly high compared to current knowledge of multiple sexual partnerships. One study suggested that there is a general consensus among AA men that it is the norm to have more than one sexual partner and that it is acceptable for men to have concurrent partners [12]. Other studies have suggested that drug use, the club environment, peer pressure, and lack of trust in, and fear of loss of confidentiality within, health care systems as major negative factors for both screening and treatment of common STIs $[13,14]$. Ensuring confidentiality can play a key role in encouraging teens and young adults to seek STI diagnosis and treatment [15]. Our study findings suggest confidentiality a major barrier to both screening and treatment of STIs. It may be harder for women to negotiate safe sex and ask for condoms to be used, which was reflected in a participant's statement: "As a female, I don't feel it's appropriate to initiate condom use prior to sex if the male doesn't have a condom." Another study reported that females are not comfortable to initiate the condom use [16].

To avoid common STIs, individuals must have monogamous sexual partners, increased understanding of how STIs are transmitted, increased access to screening, and availability of condoms $[17,18]$. In addition to these factors, our study suggested that abstinence, proper use of condoms, and staying away from the club environment were ways to avoid STIs.

The most important limitation of the study is that the findings are from a small sample of focus group participants. The results need to be confirmed by conducting a larger study with a representative sample. There is also a potential of selection bias in the recruitment of focus group participants. We used trained community leaders and potential participants for the recruitment of final focus group participants, who met the study selection criteria. Evidence from an earlier study suggests that the method utilized by this study reduces under sampling of reluctant individuals, and volunteer bias due to enthusiastic and cooperative responders [19].

Due to the sensitive nature of the subject and the stigma associated with STIs, this study may not have achieved adequate depth and breadth of responses. A more rigorous design including focus groups and in-depth one-on-one interviews would result in more complete information. Nonetheless, findings from the study are important for the next steps to address the epidemic of STIs in this community.

\section{Appendix}

Focus group questions to lead discussion and to guide facilitators.

Part I. STD risk factors, testing, and treatment (50 $\mathrm{min})$.

(1) STDs in the community (10 min).

(a) How do you see STDs in the (North) Omaha community? 
(i) How big is the problem? please explain

(ii) Do many people in Omaha have STDs?

(2) STD risk factors (20 min).

(a) What are the risk factors for STDs?

(i) What are reasons that some people get STDs? Example: individual's age, multiple partners

(b) What keeps people from getting STDs?

(i) If we want to avoid getting STDs, what can we do?

Example: use of condoms

(c) What conditions are likely to be associated with STDs?

(i) Examples: Social, environmental, and behavioral

(3) STD testing and treatment (20 min).

(a) Where do people go to get STD testing and treatment in (North) Omaha?

(i) Do you know a place where people can get STD testing and/or treatment in (North) Omaha?

(ii) If participants say that there is no place in North Omaha, ask where else they go

(b) Why do not some people get tested for STDs?

(i) How comfortable do you (and your friends) feel about going for STD testing?

(ii) What makes you or your friends uncomfortable about getting STD test?

(c) Why don't some people get treatment for STDs?

(d) What ideas do you have to increase testing and treatment for STD in North Omaha?

Part II. Opinion and recommendations about data collection protocol (20 min).

One of the goals of this project is to get an accurate picture about the burden of STD infection in the community. For example, it will be helpful to know what percentages of people living in the community are actually affected by STDs. This will help to understand the extent of the problems the community is experiencing. Also, it will serve as the baseline to see how the trend will change in future. If and when we start a new program to address STD issues, we can tell whether the program helps to decrease the STD problems. That is why the baseline information is needed.

There are different ways to collect information about STD infection in the community. One is to ask people whether they are affected or not. This approach has a problem because many people with STDs have no symptoms and many people have never been tested. So, in order to get accurate information, it is helpful to test for STD.

We need your suggestions and input about how the following approach may or may not work. We value your opinions and want to make sure the testing is done in a respectful and acceptable way. In order to find out the overall percentage of people affected by STDs, a representative sample of our community residents needs to be found. Ideally, it would be good to get information from people who live in various areas within the community. That way the result will be more representative.

There are different ways to do testing. Here are a few examples.

(1) Set up STD testing at different neighborhood events and/or gatherings such as at the community centers, library, basketball play, valentine day dance party, and so forth.

(2) A trained staff member will visit houses in the community to offer testing.

(3) Offer STD testing at the neighborhoods clinics or at a doctor's office.

(4) If we want to know the accurate picture of STDs in our community, which idea would work the best? Do you have other suggestions?

\section{Acknowledgments}

The funding for this study came from the Dean's Mentored Research Program of the $\mathrm{CoPH}$, UNMC. There was no financial or other form of conflict of interests to any of the authors. The study was approved by the IRB of UNMC (IRB\# 444-10-FB).

\section{References}

[1] Centers for Disease Control and Prevention, "STD Trends in the US: 2010 National Data for Gonorrhea, Chlamydia, and Syphilis," 2010, http://www.cdc.gov/std/stats10/trends.htm.

[2] Douglas County Health Department, "STDs in Douglas County," 2012, http://www.douglascountyhealth.com/images/stories/ stats/morbidity/STD2011_short2011\%20Census\%20Estimtate. pdf.

[3] D. Heymann, Control of Communicable Diseases Manual, American Public Health Association, Washington, DC, USA.

[4] W. Cates Jr. and J. N. Wasserheit, "Genital chlamydial infections: epidemiology and reproductive sequelae," American Journal of Obstetrics and Gynecology, vol. 164, no. 6, pp. 1771-1781, 1991.

[5] "WHO Task Force on the Prevention and Management of Infertility. Tubal infertility: serologic relationship to past chlamydial and gonococcal infection. World Health organization Task Force on the Prevention and Management of Infertility," Sexually Transmitted Diseases, vol. 22, pp. 71-77, 1995.

[6] Centers for Disease Control and Prevention, "STDs \& Pregnancy-CDC Fact Sheet," 2011, http://www.cdc.gov/std/pregnancy/STDFact-Pregnancy.htm.

[7] Centers for Disease Control and Prevention, "The Role of STD Detection and Treatment in HIV Prevention," 2011, http://www.cdc.gov/std/hiv/stdfact-std\&hiv.htm.

[8] J. Kmodel, "Focus Groups as A Qualitative Method for CrossCultural Research in Social Gerontology," 1995, http://deepblue. lib.umich.edu/bitstream/handle/2027.42/10823_2004_Article_ BF00972029.pdf;jsessionid=08E68466E68A1AC484EAC1B1E $5 \mathrm{E} 73476$ ? sequence $=1$. 
[9] R. Magnani, K. Sabin, T. Saidel, and D. Heckathorn, "Review of sampling hard-to-reach and hidden populations for HIV surveillance," AIDS, vol. 19, no. 2, supplement, pp. S67-S72, 2005.

[10] B. Lichtenstein and L. H. Bachmann, "Staff affirmations and client criticisms: staff and client perceptions of quality of care at sexually transmitted disease clinics," Sexually Transmitted Diseases, vol. 32, no. 5, pp. 281-285, 2005.

[11] E. C. Tilson, V. Sanchez, C. L. Ford et al., "Barriers to asymptomatic screening and other STD services for adolescents and young adults: focus group discussions," BMC Public Health, vol. 4, article 21, 2004.

[12] M. P. Carey, T. E. Senn, D. X. Seward, and P. A. Vanable, "Urban African-American men speak out on sexual partner concurrency: findings from a qualitative study," AIDS and Behavior, vol. 14, no. 1, pp. 38-47, 2010.

[13] A. Uusküla, K. Kangur, and L. A. McNutt, "Barriers to effective STI screening in a post-Soviet society: results from a qualitative study," Sexually Transmitted Infections, vol. 82, no. 4, pp. 323326, 2006.

[14] C. Oberg, M. Hogan, J. Bertrand, and C. Juve, "Health care access, sexually transmitted diseases, and adolescents: identifying barriers and creating solution," Current Problems in Pediatric and Adolescent Health Care, vol. 32, no. 9, pp. 320-339, 2002.

[15] "Help teens stay safe: identify barriers to prevent pregnancy, STD infection," Contraceptive Technology Update, vol. 19, pp. 69-71, 1998.

[16] C. Cook, “Nice girls don't': women and the condom conundrum," Journal of Clinical Nursing, vol. 21, pp. 535-543, 2012.

[17] K. M. Stone, "Avoiding sexually transmitted diseases," Obstetrics and Gynecology Clinics of North America, vol. 17, no. 4, pp. 789799, 1990.

[18] D. Henning, A. Ryan, L. Sanci, and T. Dunning, "Screening for chlamydia trachomatis: barriers for homeless young people," Australian Journal of Advanced Nursing, vol. 24, no. 3, pp. 8-13, 2007.

[19] L. Platt, M. Wall, T. Rhodes et al., "Methods to recruit hard-toreach groups: comparing two chain referral sampling methods of recruiting injecting drug users across nine studies in Russia and Estonia," Journal of Urban Health, vol. 83, no. 7, pp. i39-i53, 2006. 


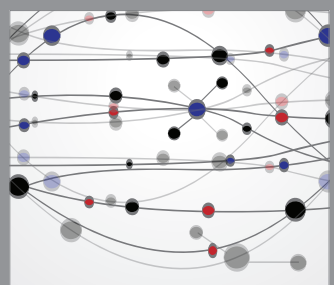

The Scientific World Journal
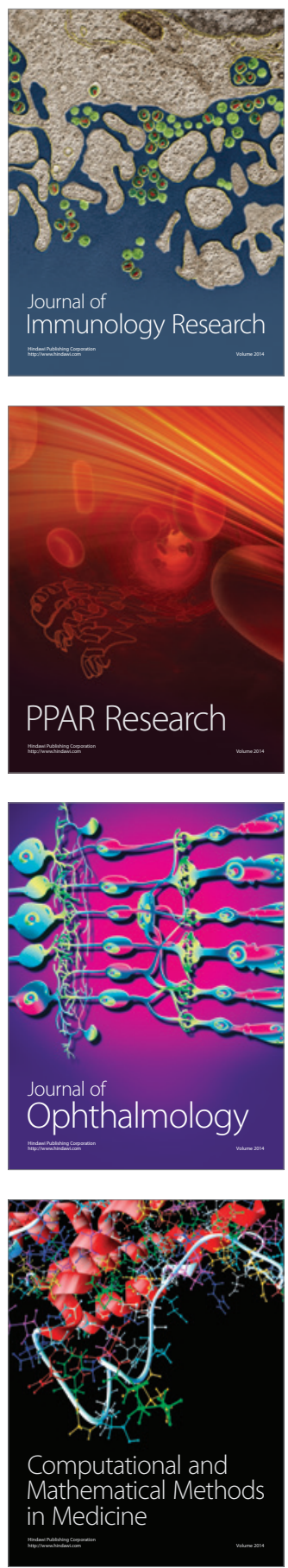

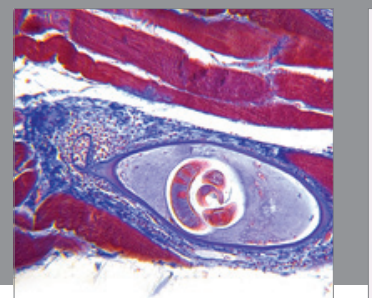

Gastroenterology

Research and Practice
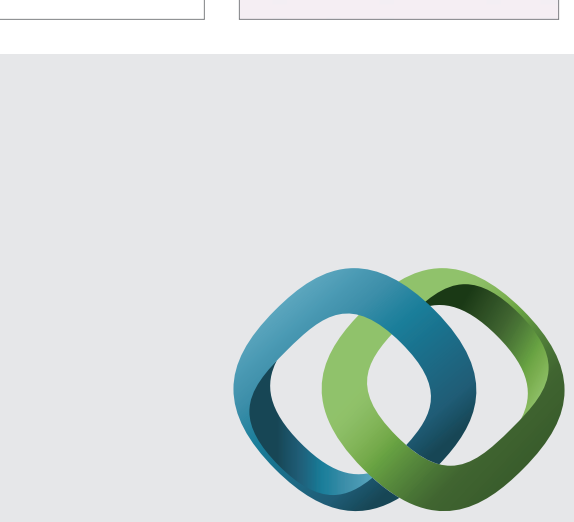

\section{Hindawi}

Submit your manuscripts at

http://www.hindawi.com
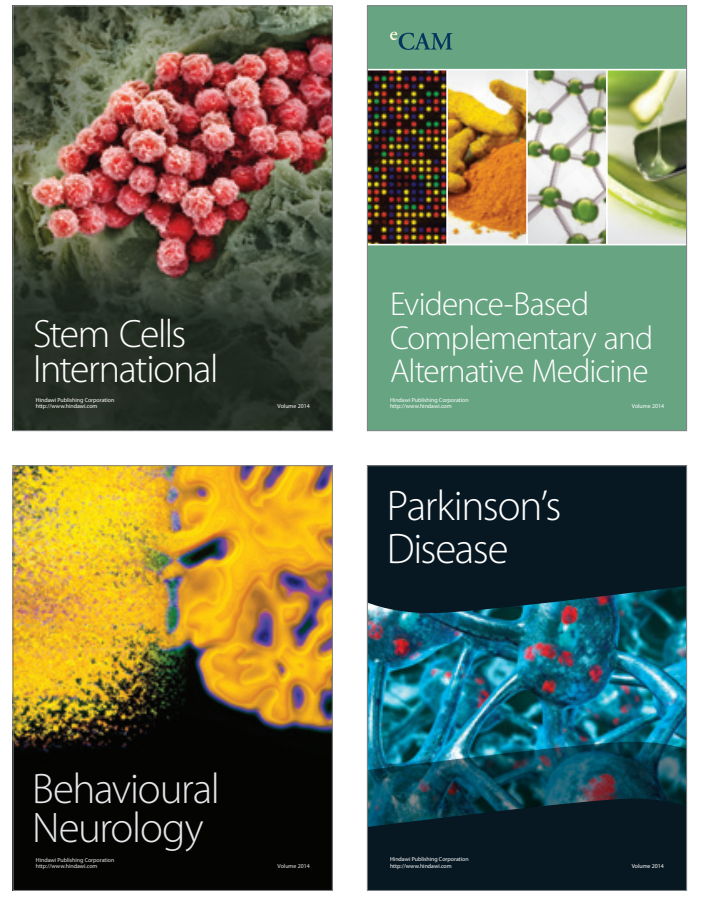
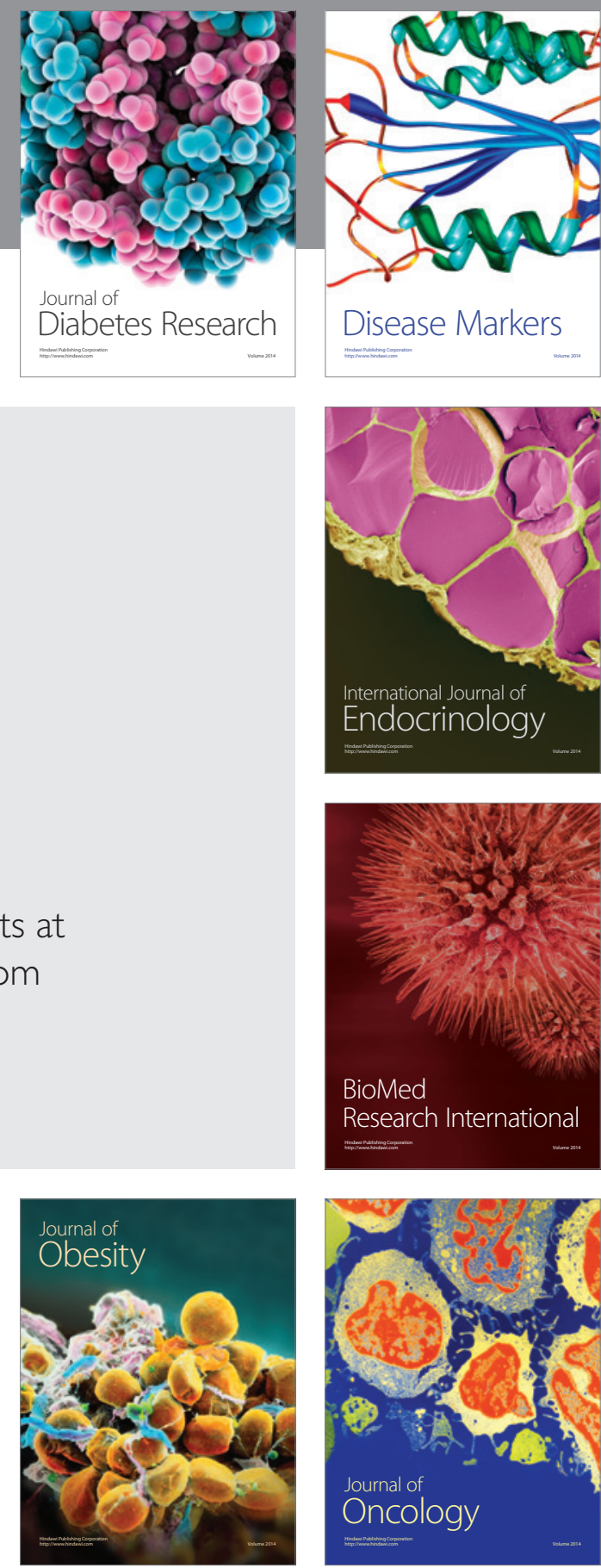

Disease Markers
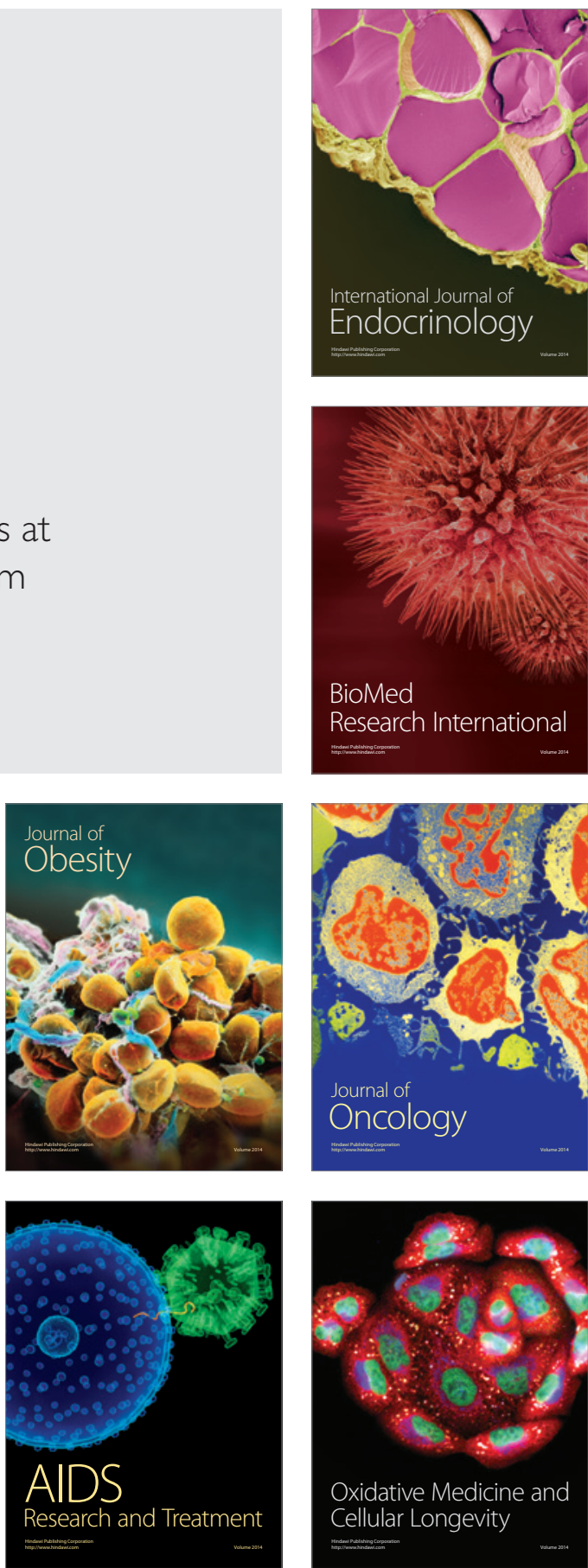